\title{
The Determination of Lattice Parameters Using Single EBSD Patterns
}

\author{
Lili $\mathrm{Li}^{1}$, Ming $\mathrm{Han}^{1}$, and Gert Nolze ${ }^{2}$ \\ 1. East China Jiaotong University, Nanchang, China \\ 2. Federal Institute for Materials Research and Testing (BAM), Berlin, Germany
}

Usually, an electron backscatter diffraction (EBSD) pattern displays dozens of well visible Kikuchi bands which form hundreds of intersections. They represent crystallographic zone axes [uvw], whereas the connections between them define the traces of the diffracting lattice planes (hkl). On the other hand, all (hkl) of a single zone are part of a lattice plane in reciprocal space with the indexing [uvw]. Direction and distance of a reciprocal lattice point hkl from the origin are determined by slope and width of the respective Kikuchi band. Unfortunately, EBSD patterns always suffer from gnomic distortions [1] so that respective corrections are necessary. In addition, caused by the complex profile and the typically small thickness, band-widths measurements have a relative error of up to $20 \%$ [2]. Thus, an EBSD pattern provides abundant crystallographic information but is assumed to deliver a disappointingly low accuracy and precision in lattice parameters.

In general, the precision of a quantity can be improved by consideration of a higher number of measurements. To this end a computer program (EBSDL) has been developed in order to consider as many as possible bands and zone axes, and increase the precision of the resulting lattice parameters. A graphical user interface supports the manual definition of bands and the control of the refinement procedure. EBSDL derives the lattice parameters from a single EBSD pattern without any additional information about crystal symmetry. Only the accelerating voltage, the approximate position of the pattern center PC, and the sample-detector distance DD are required where the latter will be optimized during refinement.

In order to exclude uncertainties regarding the electron wavelength and lattice parameters, a simulated diffraction pattern [3] of calcite $\left(\mathrm{CaCO}_{3}, a=0.499 \mathrm{~nm}\right.$ and $c=1.706 \mathrm{~nm}$, trigonal [4]) will be used as example. After manual detection of well visible band positions and widths, EBSDL derives the Bravais lattice type as well as the lattice parameters. Figure 1 displays the interface of $E B S D L$ including the loaded EBSD pattern. The over-laid result dialog shows that the loaded pattern matches a phase with hexagonal translation lattice. The derived lattice parameters are $a=0.497 \mathrm{~nm}$ and $c=1.688 \mathrm{~nm}$. Compared to the input data used during pattern simulation, relative errors of about $0.4 \%$ for $a$, and $1.06 \%$ for $c$ result. For the lattice parameter ratio a value of $c / a=0.7 \%$ can be calculated.

Analyzing dozens phases of different symmetry, the typical error for lattice parameters is $<2.5 \%$. The main limitation is that lattice parameters are derived from the band widths which are very narrow and therefore characterized by a huge error, see above. However, according to [2] the error of lattice parameter ratios is often clearly smaller than $1 \%$. This is based on the exclusive use of the zone axes which are a) more numerous than bands, b) more accurate in positioning, and c) have bigger distances to each other so that measurement errors are smaller, relatively. More precise values result if PC and DD are exactly known and not part of the refinement. 
In comparison to approaches based on HOLZ rings [5] the presented software works for all patterns containing a sufficient number of bands, so that $E B S D L$ endows scanning electron microscopy with the feasibility of a direct determination of lattice parameters for unknown phases.

\section{References:}

[1] K Baba-Kishi, Scanning 20 (1998), p. 117.

[2] D Dingley and S Wright, J. Appl. Cryst. 42 (2009), p. 234.

[3] A Winkelmann et al, Ultramicroscopy 107 (2007), p. 414.

[4] R Reeder, Rev. Mineral. Geochem. 11 (1983), p. 1.

[5] J R Michael and R P Goehner, Micr. Microanal. 6.2 (2000), p. 946.

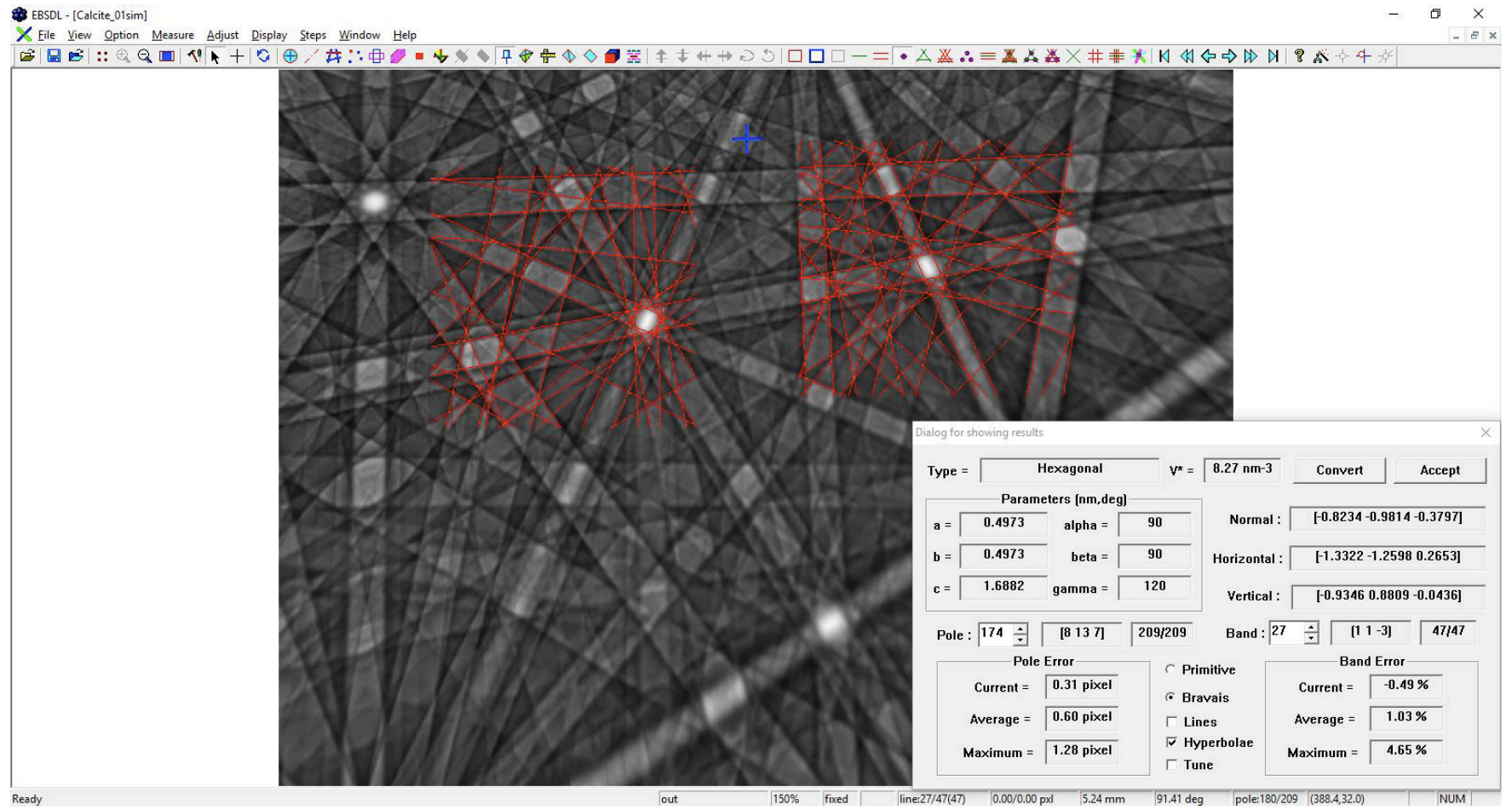

Figure 1. The image shows a screen copy of the interface of the program EBSDL with a simulated EBSD pattern of calcite. The blue cross displays the refined pattern center po-sition, and the red hyperbolae reflect the determined band edges. In order to improve the comparison between derived hyperbolae positions and band edges, only two regions of the entire pattern are selected.

The result dialog in the lower left corner presents the solution as well as the current, av-erage and maximum errors of the bands and poles. As the mouse moves on the pattern, band and zone axis indices nearest to the cursor position are displayed. Additionally, cor-responding error information can be instantly updated in the result dialog, and the ordinal number of the nearest band and zone axis will be shown in the panes of the status bar. 